\title{
Rebamipide in Functional and Organic Dyspepsia: Sometimes the Best Offense Is a Good Defense
}

\author{
Vikram Rangan ${ }^{1} \cdot$ Filippo Cremonini ${ }^{2}$
}

Published online: 20 February 2018

○) Springer Science+Business Media, LLC, part of Springer Nature 2018

Dyspepsia is a very common complaint in gastroenterology (GI) and primary care clinics, affecting approximately one fifth of the global population. While the past decade has seen advances in defining and in appropriately characterizing dyspepsia and its subtypes, effective treatments for this condition remain elusive, particularly for patients whose dyspepsia is of functional (FD), rather than of structural or demonstrable (organic) cause. The most recent American College of Gastroenterology (ACG) guidelines [1] recommend sequential trials of proton pump inhibitor (PPI), tricyclic antidepressants, prokinetic drugs, and psychologic therapies for patients without organic cause for their symptoms, or with symptoms not responding to appropriate treatment of the underlying organic cause (such as eradication of $\mathrm{H}$ pylori infection). None of these approaches, however, are supported by high-quality scientific evidence. The development of new treatment strategies for dyspepsia still represents a major unmet clinical need.

In this issue of Digestive Diseases and Sciences, Jaafar et al. [2] demonstrate that rebamipide, a drug used mostly in Asia and Russia intended to enhance foregut mucosal defense mechanisms such as mucus and bicarbonate secretion, improved symptoms in individuals with dyspepsia. The authors performed a rigorous meta-analysis that included 17 studies comparing rebamipide, administered with or without antisecretory agents, to placebo, to a comparator medication, or to no treatment. Their analysis included three studies comparing the combination of rebamipide plus antisecretory therapy with antisecretory therapy alone. The authors also differentiated individuals with organic dyspepsia such

Filippo Cremonini

filcrem@gmail.com

1 Division of Gastroenterology, Department of Medicine, Beth Israel Deaconess Medical Center, Harvard Medical School, Boston, MA, USA

2 Las Vegas Gastroenterology, First Class Medicine, 2910 S Maryland Pkway Ste. 9B, Las Vegas, NV 89119, USA as nonsteroidal anti-inflammatory drug (NSAID)-induced gastropathy or ulcers, reflux esophagitis, or $\mathrm{H}$. pyloriassociated ulcer, from those with FD. The authors analyzed 1224 subjects who received rebamipide and 946 subjects who received placebo or control medications. Symptom improvement (when reported as a binary outcome) was noted among patients with organic dyspepsia. This metaanalysis reports also an overall reduction in mean upper GI symptom scores across all patients, and noted similar symptom improvements across patients with organic and FD. A greater improvement in symptoms appeared to be related to longer treatment duration. Notably, 15/17 studies from this meta-analysis were conducted in East Asia, whereas two were conducted in Western populations.

Multiple proposed mechanisms for FD exist, including delayed gastric emptying, inadequate fundic accommodation to meals, abnormal central pain processing, and anxiety or other psychiatric comorbidity [3]. There has also been a recently demonstrated association between FD and both duodenal eosinophilia [4, 5], and duodenal eosinophil degranulation [6]. An evolving paradigm posits that the recruitment of excess eosinophils (possibly in response to prior infection or allergen exposure) leads to degranulation and local nerve damage, predisposing patients to pain, increased mucosal permeability, and altered motility [7].

Rebamipide purportedly activates cyclooxygenase, enhancing prostaglandin release while inhibiting inflammatory cytokines and scavenging free radicals [2]. Prostaglandins are among the key mediators at the epithelial level that protect the gastric mucosa from injury, along with multiple pre-epithelial, epithelial, and microvascular factors [8]. Mechanistically, it would appear logical that an agent that releases prostaglandins with consequent mucosal protection would improve symptoms that are presumably due to acid injury or to weakened defense mechanisms such as peptic ulcer disease or NSAID-induced gastropathy. Prostaglandin release would also protect and favor symptom recovery among patients with chronic gastritis not associated with 
NSAIDs. The effect of rebamipide on symptoms among those patients with no evidence of gastric or duodenal mucosal abnormality, however, is particularly intriguing, raising several explanatory hypotheses: First, a minimalmoderate amount of gastroduodenal mucosal injury could still be present in individuals with FD. While underreported, such structural change could account for the modest, though significant effect seen with rebamipide. Second, cytokine release due to eosinophil degranulation may mediate both gastroduodenal dysfunction and extra intestinal effects (including central symptoms, such as anxiety) in patients with FD [7]. Rebamipide-related impairment of inflammation due to cytokine release may constrain this sequence of events, thereby providing symptom relief.

As with most meta-analyses, multiple limitations exist. For example, this study attempts to combine data from a heterogeneous group of patients. Symptom improvement was measured using multiple scales and often was not the primary endpoint, further increasing the heterogeneity and diminishing the interpretability of the study results.

Improvement of FD due to rebamipide was seemingly superior in Asian patients. A key question is whether there are different drivers of dyspepsia in Asian and non-Asian populations. Since the Western study populations were unfortunately very small, the data did not offer further insights into the cause of the apparent discrepancy. Further, rebamipide was more effective in $H$. pylori infected dyspepsia patients [9]. Testing for $H$. pylori, and offering eradication if positive, is generally the first-line strategy in dyspepsia management in the USA, a recommendation supported by high-quality evidence [1]. Eradication of $H$. pylori also appears to have a greater clinical impact in Asian patients. While it is unlikely that the use of a mucosal protective agent such as rebamipide would be recommended for dyspepsia management prior to $H$. pylori treatment, an important area worthy of further investigation would be to assess whether rebamipide is associated with dyspepsia resolution and improvement in those patients with persistent symptoms despite successful $H$. pylori eradication.

Overall, the evidence offered for the use of rebamipide in organic dyspepsia was more robust than for the management of FD. As management of organic dyspepsia generally entails treatment or healing of the structural or organic cause prior to the institution of therapies primarily directed at symptom management, further study of the efficacy of rebamipide in patients with organic dyspepsia that persists after treatment of an underlying organic cause holds value for its potential clinical use.

Gastroenterologists practice in an era of concern about untoward medication side effects. Recommended treatment steps for FD are generally based on off-label use [1]. Using the example of PPIs, which are commonly used in FD and are recommended in clinical guidelines, one can argue that the benefit/risk balance for their long-term use in FD might not be as robust as it is for other structural conditions of the upper GI tract [10]. Cost consideration is also paramount in the approach to a chronic condition such as FD. Research and data on additional approaches, possibly devoid of significant adverse effects and of lower cost is welcome.

Ultimately, this noteworthy study provides evidence that rebamipide is efficacious in the management of FD and organic dyspepsia, suggesting a biologically plausible mechanism-of-action for such effects. Though many unanswered questions remain, the meta-analysis of Jaafar and colleagues raises interest with regard to a possible new treatment modality that uses a traditional approach focused on mucosal protection for FD, an overwhelmingly common condition with unmet therapeutic needs.

Author contributions VR helped in planning and drafting of the manuscript. FC involved in planning, critical revision of the manuscript for important intellectual content and final approval of the version to be published.

\section{Compliance with Ethical Standards}

Conflict of interest The authors declare that have no conflicts of interest.

\section{References}

1. Moayyedi PM, Lacy BE, Andrews CN, Enns RA, Howden CW, Vakil N. ACG and CAG clinical guideline: management of dyspepsia. Am J Gastroenterol. 2017;112:988-1013.

2. Jaafar MH, Safi SZ, Tan M-P, et al. Efficacy of rebamipide in organic and functional dyspepsia: a systematic review and meta-analysis. Dig Dis Sci. 2018 (Epub ahead of print). https://doi.org/10.1007/ s10620-017-4871-9.

3. Talley NJ, Ford AC. Functional Dyspepsia. $N$ Engl J Med. 2015;373:1853-1863.

4. Talley NJ, Walker MM, Aro P, et al. Non-ulcer dyspepsia and duodenal eosinophilia: an adult endoscopic population-based case-control study. Clin Gastroenterol Hepatol. 2007;5:1175-1183.

5. Walker MM, Aggarwal KR, Shim LS, et al. Duodenal eosinophilia and early satiety in functional dyspepsia: confirmation of a positive association in an Australian cohort. J Gastroenterol Hepatol. 2014;29:474-479.

6. Du L, Shen J, Kim JJ, Yu Y, Ma L, Dai N. Increased duodenal eosinophil degranulation in patients with functional dyspepsia: a prospective study. Sci Rep. 2016;6:34305.

7. Fan K, Talley NJ. Functional dyspepsia and duodenal eosinophilia: a new model. J Dig Dis. 2017;18:667-677.

8. Laine L, Takeuchi K, Tarnawski A. Gastric mucosal defense and cytoprotection: bench to bedside. Gastroenterology. 2008;135:41-60.

9. Talley NJ, Riff DS, Schwartz H, Marcuard SP. Double-blind placebo-controlled multicentre studies of rebamipide, a gastroprotective drug, in the treatment of functional dyspepsia with or without Helicobacter pylori Infection. Aliment Pharmacol Ther. 2001;15:1603-1611.

10. Freedberg DE, Kim LS, Yang YX. The risks and benefits of longterm use of proton pump inhibitors: expert review and best practice advice from the American gastroenterological assocciation. Gastroenterology. 2017;152:706-715. 